\title{
Spatio-Temporal Gait Analysis Based on Human-Smart Rollator Interaction
}

\author{
Atia Cortés ${ }^{1[0000-0002-4394-439 X]}$, Antonio B. Martínez ${ }^{2[0000-0003-0414-7520]}$, and \\ Javier Béjar ${ }^{1[0000-0001-5281-3888]}$ \\ 1 KEMLg, Universitat Politecnica de Catalunya - Barcelona Tech, Spain \\ \{acortes, be jar\} @ cs. upc. edu https://kemlg.upc.edu/en \\ 2 GRINS, Universitat Politecnica de Catalunya - Barcelona Tech, Spain \\ antonio.b.martinez@upc.edu
}

\begin{abstract}
The ability to walk is typically related to several biomechanical components that are involved in the gait cycle (or stride), including free mobility of joints, particularly in the legs; coordination of muscle activity in terms of timing and intensity; and normal sensory input, such as vision and vestibular system. As people age, they tend to slow their gait speed, and their balance is also affected. Also, the retirement from the working life and the consequent reduction of physical and social activity contribute to the increased incidence of falls in older adults. Moreover, older adults suffer different kinds of cognitive decline, such as dementia or attention problems, which also accentuate gait disorders and its consequences. In this paper we present a methodology for gait identification using the on-board sensors of a smart rollator: the $i$-Walker. This technique provides the number of steps performed in walking exercises, as well as the time and distance travelled for each stride. It also allows to extract spatio-temporal metrics used in medical gait analysis from the interpretation of the interaction between the individual and the $i$-Walker. In addition, two metrics to assess users' driving skills, laterality and directivity, are proposed.
\end{abstract}

Keywords: Assistive technologies $\cdot$ Healthcare $\cdot$ Gait analysis

\section{Introduction}

In nowadays greying society, a large amount of people require appropriated and personalized assistance, This circumstance leaves room for new technologies that offer this population an extraordinary opportunity to perform their activities of daily living (ADL) and to improve their autonomy. Of all world regions, Europe has the highest proportion of population aged 65 or over, a statistic that becomes more pessimistic according to the baseline projection of Eurostat, which shows that this percentage will almost double to more than $25 \%$ in the year 2050 [17].

In addition, in this population sector the frequency of falls increases with age and frailty level. These to factors are a main cause of unintentional injury, due to a combination of biological factors and disease-related conditions. This has several implications in the Quality of Life (QoL) of the elderly: the more they reduce their ADLs, the more their frailty and fear of falling increases, losing their residual skills as a consequence.

Cortés, A.; Martínez, A.; Béjar, J. Spatio-temporal gait analysis based on human-smart rollator interaction.

"Communications in computer and information science", 2019, vol. 1002, p. 68-83.

The final authenticated version is available online at https://doi.org/10.1007/978-3-030-16785-1_6 
This will represent a challenge for the public health systems that will have to face a huge economic impact to deal with this demographic situation. This is not sustainable in certain countries and it is already a world-wide issue.

Assistive Technologies (AT) play a key role in providing solutions to improve the QoL of this target population. Furthermore, one of the main objectives for the European Union H2020 programme has been to focus on the analysis of the causes and consequences of pathologies in order to find patterns that will support early detection of a disease or associated risks. Consequently, the care community could take decisions on preventive intervention and educational information in order to delay the physical or cognitive decline of the elderly and try to keep them independent as long as possible living in the community. The evolution of ICT tools in collaboration with medical knowledge has empowered the use of sensorized assistive devices that can provide ubiquitous, real-time information about a person's evolution in terms of physical and cognitive conditions.

In this paper we introduce a set of metrics to assess gait quality and risk of falling in elderly individuals using a smart rollator: the $i$-Walker [6]. The aim is to identify significant characteristics, providing in a future a decision support tool to clinicians and caregivers for walking assessment and user profiling.

\subsection{Plan of Work}

In Section 2 the context in which the $i$-Walker was used to collect the data from a group of elderly people is detailed. Section 3 introduces a set of definitions and representations that will be used to measure users' performance while using the $i$-Walker. Section 4 explains the methodology applied to the signals collected from the sensors of the walker to extract gait information. In section 5 some results from the data collected from two groups of elder adults are depicted. Finally section 6 provides some conclusions and future work.

\section{Components of the study}

This section describes the different components that were used to carry on the study here presented: the measurement tool, the $i$-Walker, the participants and the type of exercises that were performed.

\subsection{Measurement Tool: the $i$-Walker}

Assistive devices are developed to help in the daily lives of elderly and disabled people. The $i$-Walker is based on a standard 4-wheeled rollator with embedded sensors and actuators. Its aim is to provide mobility support and rehabilitation to persons with a physical and/or cognitive disability, as well as to monitor their activities. It has a distributed micro-controller architecture which drives the system and records and provides information to clinicians (see Figure 1): 
1. Handlers contain force sensors which measure pushing, leaning and lateral forces ( $X, Y$ and $Z$ axis respectively) on both hands. They also have a multicoloured lighting ring (5) used to indicate different states of the $i$-Walker to the user, like calibration status and battery level.

2. Rear wheels embed motors that work as actuators, the purpose is to supply a set of operative modes which provide pushing or pulling help depending on the control strategy.

3. Central box under the seat contains the computing power on-board (e.g. a Raspberry $\mathrm{Pi}$ ) and a set of sensors that will provide information about movement and tilt.

4. Blocking brakes are situated in the rear legs and provide a braking help when the $i$-Walker detects the user is driving in downhill. For safety reasons the $i$-Walker automatically stops when the user releases the handlers, that is, when no forces are detected on them.
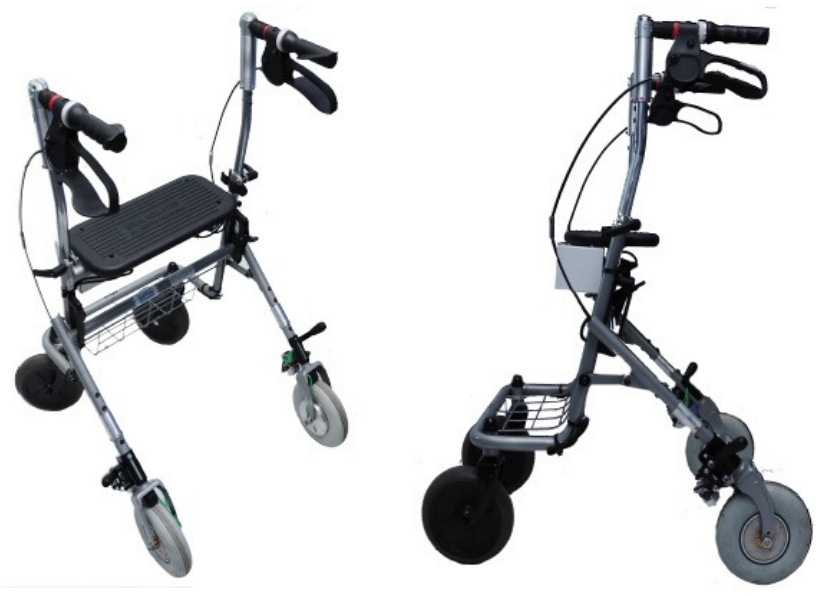

Fig. 1. The $i$-Walker

For each exercise or activity that users perform with the $i$-Walker, data from each sensor is recorded every $100 \mathrm{~ms}$ and stored in the Raspberry Pi as individual time series. From its on-board sensors, the $i$-Walker can infer the travelled distance, the walking time, the maximal speed, the maximum of the lateral deviation and the force exerted individually in both handlebars. Moreover, after processing the signals, it is possible to obtain specific information of the user's walking behaviour at a given moment of the exercise, being either in terms of distance or time in the trajectory, a concrete step number or by maxima speed or force. 


\subsection{Walking Tests}

The 10 Metre Walking Test (10MWT) is a performance measure used in rehabilitation to assess walking speed over a short distance. It can be employed to determine functional mobility, gait and vestibular function [1].

During a 10MWT exercise, the subject has to walk 10 metres in a maximal straight way. This is traditionally measured with a stopwatch in terms of the time duration and the resulting average speed of the participant. The first and last two metres, that are related to the acceleration and deceleration phases, are discarded and only the central part of the exercise is taken into account, where it is assumed that the walking speed is almost constant. It is considered that gait speed can be a useful outcome to assess the physical decline of elderly people as well as to predict the risk of falling, other health deterioration or even survival [8]. Gait velocity has been related with the ageing process, although results are uncertain due to biological and behavioural differences among the population [13].

Another commonly used metric is the 6 Minutes Walking Test $(6 \mathrm{mWT})$, which assesses endurance in older adults by measuring the walked distance in that time. In terms of gait analysis and walking behaviour patterns, this measure should be more relevant since it provides more knowledge about each individual. For this study, it was decided to reduce this walking time to only three minutes following the clinical team indications. Thus, the participants walked in a 40-metres indoor corridor during three minutes. When the end of the corridor was reached, they turned back and walked to the starting point. Afterwards, the turning phases were excluded in the pre-processing step, storing each straight line as a single exercise. Moreover, in order to be compliant with the 10MWT exercise, the initial and final $20 \%$ were excluded. Then the average walking speed is calculated for the central $60 \%$ of the exercise. Consequently, bounds are recalculated, keeping the piece of exercise that starts and ends at this average walking speed.

\subsection{Participants}

Our initial target population consists of older adults, aged 65+ years old, with a good cognitive condition (MMSE $>23$ ) and having suffered a previous fall in the last year or being considered as a person with high risk of falling (Tinetti Scale < 21 [14]). Individuals with any diagnosed mental disorder, such as dementia, or bearers of implantable electronic devices were excluded. Participants involved in this study come from residential care centres and hospitals from Italy and Spain and are divided in two different groups.

The first group is composed by 85 participants of the European funded project $I$ DONT FALL (IDF from now on, [7]), which has already been introduced in previous works $[4,3]$. In addition, data from another 60 participants from a care centre in Madrid $(M A D)$ involved in the IDF project were collected following similar inclusion and exclusion criteria. People in this first group performed the 10MWT in an indoor corridor twice. In the case of $I D F$, data was collected before and after a three months training program ( $\mathrm{T} 0$ and $\mathrm{T} 1$ periods respectively), where participants were assigned to a physical, cognitive, mixed or placebo group. The aim was to prove the effectiveness of a 
Table 1. Participants' age and gender distribution for each pilot. Each column represents a pilot.

\begin{tabular}{|l|l|l|l|}
\hline \multicolumn{1}{|c|}{ Pilot } & IDF $(\mathrm{N}=85)$ & MAD $(\mathrm{N}=59)$ & CVI (N = 41) \\
\hline Age Range & $0(0 \%)$ & $0(0 \%)$ & $17(41 \%)$ \\
Y & $35(41 \%)$ & $8(13 \%)$ & $9(21 \%)$ \\
M & $50(59 \%)$ & $51(87 \%)$ & $16(38 \%)$ \\
O & Age & & \\
Mean ( \pm SD) & $82.7( \pm 8.3)$ & $87.6( \pm 5.8)$ & $64.1( \pm 23.1)$ \\
Min & 65 & 70 & 22 \\
Max & 97 & 96 & 94 \\
\hline Gender & $58(68 \%)$ & $37(63 \%)$ & $34(81 \%)$ \\
F & $27(32 \%)$ & $22(37 \%)$ & $8(19 \%)$ \\
M & T0 / T1 & & \\
\hline Gait Velocity & $52 / 50$ & 34 & 8 \\
Low & $27 / 22$ & 20 & 17 \\
Medium & $6 / 12$ & 4 & 16 \\
High & T0 / T1 & 2 & 25 \\
\hline Tinetti & $8 / 11$ & 20 & 9 \\
Low & $30 / 46$ & 37 & 8 \\
Medium & $47 / 28$ & 37 & \\
High & & & \\
\hline
\end{tabular}

fall prevention system by observing the reduction of the incidence of falls, along with the risk and fear of falling. The MAD group performed two 10MWT exercises on a same session. After excluding drop-outs and some cases of sensor failure, this group was composed by $28510 \mathrm{MWT}$ exercises.

The second group of participants was composed by 41 residents and workers of the Centre de Vida Independent (CVI), a care centre in Barcelona, and included people with a wider age range, under 65 years and with no previous fall. The objective was to observe differences in terms of walking behaviours among the different age groups. In this case, participants performed the $3 \mathrm{mWT}$ in a 40 metres indoor corridor, since it is able to offer a more complete profile of each user. After the data cleaning process, where the turning areas are excluded and each straight line is considered as a single exercise, this group is represented by 153 instances.

Table 1 shows the representation of the participants in each pilot by $(i)$ age $<65$ years (Y), 65-80 (M), 85>(O); (ii) gender (Female and Male); (iii) risk of falling based on the Tinetti scale (Low, Medium, High) and (iv) gait velocity, or GV (Low, Medium, High). This last metric is categorised based on [8], where authors determine that the walking speed is a relevant indicator to predict adverse events in healthy elders. Moreover, in [9], authors relate the walking speed to physical and medical status of older adults. In particular, individuals walking under $0.6 \mathrm{~m} / \mathrm{s}$ (belonging to the Low gait velocity group) are considered people at high risk of falling, along with a high probability of developing comorbidities. People walking at a gait velocity higher than $1 \mathrm{~m} / \mathrm{s}$ are categorised as High gait velocity and the rest fall in the Medium group. 


\section{Definition of metrics}

There are several methods for evaluating the human-robot interaction while driving. In our case, the $i$-Walker works with a purely reactive control and does not provide any supporting help to the user while driving. Thus, it will only act as a sensing platform that collects data during the exercise at a rate of $10 \mathrm{~Hz}$. This work focuses on the information generated from the force sensors embedded on the handles and the odometry calculated from wheel encoders.

\subsection{Spatio-Temporal Characteristics}

The ability to walk normally is related to several biomechanical components involved in the gait cycle (or stride), which is composed of the stance and swing phases. The faster we walk, the shorter the stance phase will be. Usually, when talking about walking analysis, users' performances are assessed in terms of gait quality and gate associated disturbances caused by ageing, but also to cognitive and/or physical disabilities. Different approaches on the assessment of gait quality have been proposed with different perspectives: anthropometric, spatio-temporal, kinetic or kinematic (a complete review is offered in [12]).

Gait analysis is often carried on in controlled environments, such as especial rooms with cameras that are used to identify steps or by using wearable sensors on participants. Spatio-temporal parameters provide the simplest form of objective gait evaluation in terms of time and distance. These parameters have often been used to study the relation between gait quality and cognition in older adults [9]. This paper presents a technique that uses the information extracted by the interaction of the user with the $i$-Walker, and thus with its sensors. The stride duration is measured in terms of time and distance and then related to the amount of pushing force exerted at each moment. Force sensors will not only allow to shape driving skills, but are also able to provide a qualitative spatiotemporal assessment. For each exercise, the interpretation of the $i$-Walker sensor data is able to provide the following metrics:

- walked time and distance (in seconds and metres respectively),

- average walking speed or gait velocity $(\mathrm{m} / \mathrm{s})$,

- number of strides,

- stride length distance (in metres) of a gait cycle (or the time between steps of the same foot),

- stride time duration (in seconds) of a gait cycle (or the time between steps of the same foot),

- cadence (strides/minute or the number of strides * 60 / walked time), and

- average pushing force (Newtons) of the force shape extracted from each stride

In this work, the stride length and time will be considered as the average value of the whole exercise, in addition to its standard deviation and covariance. These two parameters will define the gait variability of each user, which is also a useful indicator of gait dysfunction. 


\subsection{User Driving Skills}

There are several methods of evaluating the human-robot interaction while driving. In [15], the author proposes methods to $(i)$ analyse user driving skills and obtain user profiles according to their performance, and (ii) interpret user disagreement in a collaborative control driving strategy.

For this work, we have defined two different evaluation metrics, laterality and directivity, which measure the ability to walk directly to an objective and with small oscillations in a straight line. It will also provide information on how balanced are user's movements and force load to the $i$-Walker in relation to the deviation angle. These deviations are being considered as lateral errors and occur when the user is not driving on the desired path. These lateral errors are calculated in terms of areas (square metres). The smaller the area is, the better the driving ability. However, in a controlled exercise like the 10MWT (which is usually performed in a corridor), deviations are expected to be relatively small (less than 50 centimetres).

The first term that we have defined is the Laterality of the trajectory. Based on the 10MWT, where the user is supposed to follow a straight line but only having the starting and ending point markers as path indicators, the Laterality measures the area between the starting point and user's ending point in relation to the predetermined ending point (the final goal). An ideal straight line would always show values around zero in the $Y$-axis, while the $X$-axis would increment with the travelled distance. This metric is obtained by adding the absolute value of the area generated by the $Y$-axis for each increment in $X$-axis. In addition, this metric allows to assess body force compensation, or balance, by measuring the areas of the trajectory on the right or left side of the desired destination.

The second term defined is the Directivity of the user. Given the user's trajectory and his/her ending point (not the original goal, but the new one determined by the orientation of his/her path), we calculate how straight did he/she go to that final destination. The estimated straight line that the user is following according to his original deviation is calculated using a least squares linear regression. Not only are we representing how straight did the user go to the predetermined destination point, but also how balanced was the user's navigation, i.e. did the user drive on the left or right side of a straight (imaginary) line. The estimated line is rotated to have zero $Y$-axis values. Therefore, dimensions of the areas are proportional to this projection of the linear regression. Even though there are other possibilities to measure this metric, this rotation was made to simplify the visualisation of the areas of deviation in terms of a straight line (i.e. to simulate the ideal case where the user would follow a perfectly straight line).

\section{Methodology}

In this work, we propose a methodology for gait cycle (or stride) identification based on the interaction of the user with the $i$-Walker through the force sensors embedded in the handlers. A first proposal of this methodology was presented in [4] using the 10MWT exercises. Other researchers have also used robotic rollators and/or other automatic means to measure users' performance in the 10MWT. For instance, in [16], 
authors propose a methodology to identify steps in straight line exercises using the angular velocity. This is used to determine the swaying phase of the gait cycle when the angular velocity has zero-value. A previous methodology using the $i$-Walker was proposed in [2] using the pushing force inputs to identify gait cycles. The study was applied to a group of geriatric users with different ambulatory problems, including individuals with leg amputations. There are other approaches for gait analysis that are not using any support device, but instead use visual tools to identify steps: in [18], authors use a laser range sensor in order to track leg movements in a controlled indoor scenario; recorded kinematic data from infra-red cameras is used in [10].

It has been observed that people, and especially women, present a hip sway during the swing phase in order to balance the weight. When using a rollator, this swaying is accompanied by a pushing force coming from the arm that will allow to move it from one point to another. Hence, we can extract the movements of the individual through the $i$-Walker by using its force sensors. As shown in Fig. 2.top, left and right hand pushing forces are opposed, i.e. when one signal increases, generally the second decreases. It is then possible to interpret the number of steps performed during an exercise by using the following formula:

$$
F_{x} \operatorname{diff}=\operatorname{rhf} x-\operatorname{lhfx}
$$

where $r h f x$ corresponds to the right hand pushing force and $\operatorname{lhfx}$ is the left hand pushing force. In Fig. 2.bottom positive values on the resulting signal $F_{x} d i f f$ are those where the right hand was exerting a higher pushing force than the left hand, and thus corresponds to the steps performed by the right foot.
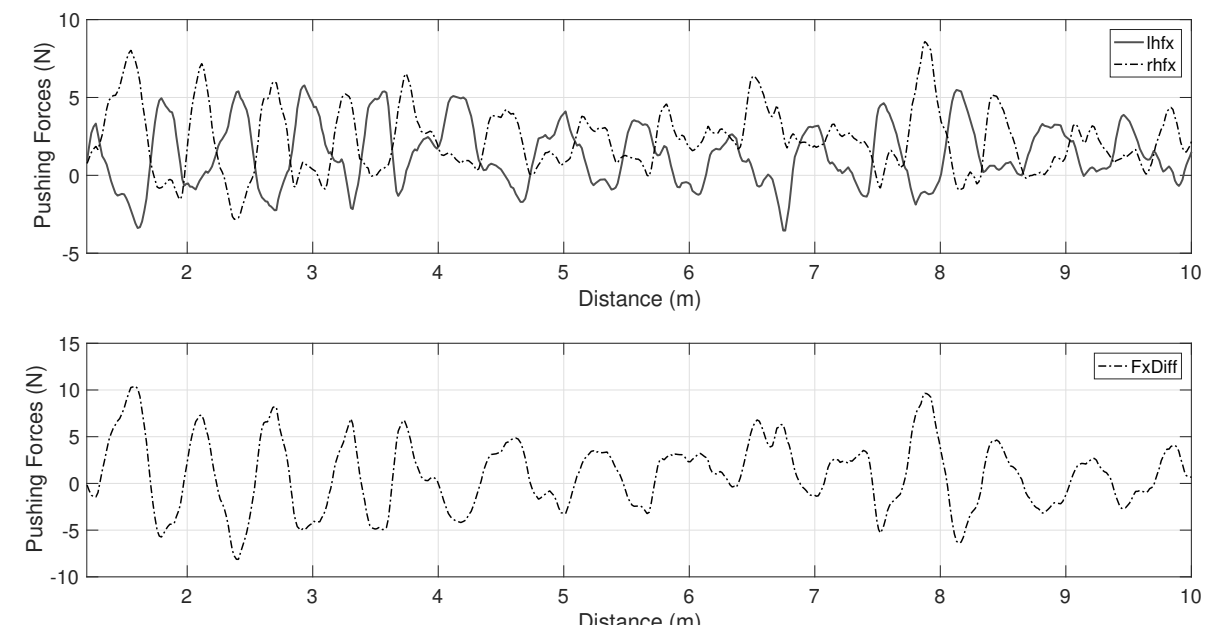

Fig. 2. top) Hand pushing force on each handlebar; bottom) Resulting pushing vector obtained with $F_{x}$ Diff 
The peaks of the signal are interpreted as gait cycles, where positive values will correspond to the right foot strides and negative values will be related to left foot strides. However, the signal needs to be filtered in order to discard smaller peaks that could be mistakenly considered as a step. The findpeaks function in MATLAB allows to identify peaks by local maxima along with different filtering options. In [4], peaks were extracted by a minimum height filter, also called prominence, based on the pushing force exerted. However, findpeaks allows also to filter by minimum distance between peaks, where distance can be either a time measure or other specified in the parameters. In this case, signals were filtered by a minimum of walked distance between strides based on the average walking speed. Figure 3 shows the final peaks considered right strides after the filtering process. Working with time series, allows to associate each peak to a moment in the exercise in terms of time and walked distance. Thus, it is possible to easily extract the spatio-temporal characteristics above mentioned for each stride and for each exercise.

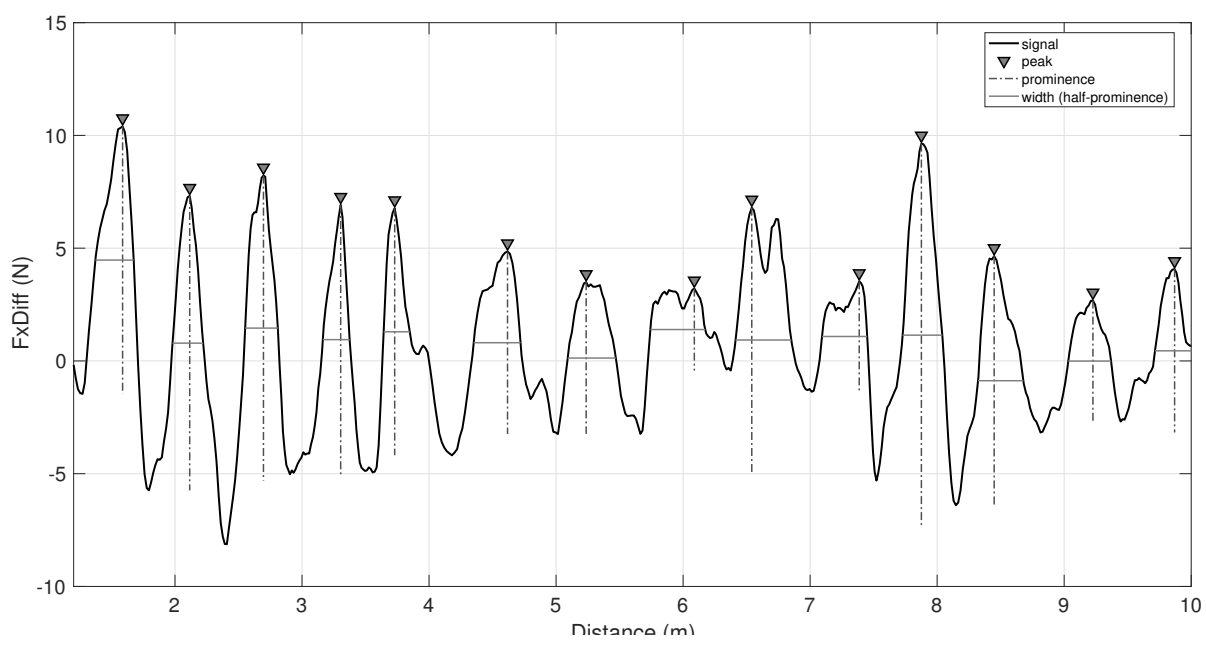

Fig. 3. Right leg strides, pushing force increments and average pushing time within peaks

As above said, the positive parts of the signal correspond to those moments where the user was exerting more force with its right hand. It is expected that in an exercise like the 10MWT, where the trajectory is assumed to be a straight line, the $F_{x} d i f f$ signal will be balanced: a person drifting to one side while driving will present less zero-time moments, since the increasings in the opposite side will not be high enough to compensate the movement. The observation of the force shape applied during the exercise illustrates the walking behaviour of each individual, i.e., how does the individual interact with the $i$-Walker and associate it with a possible physical or cognitive dysfunction.

The pushing force shape represented in Figure 3 shows that the participant starts with a secure, constant walking speed but at some point changes the pattern and presents 


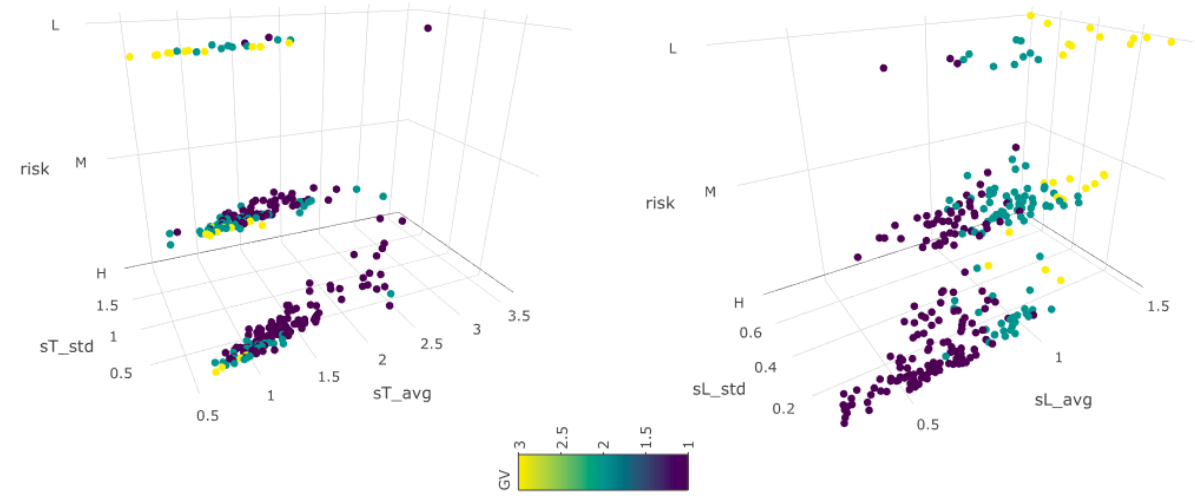

Fig. 4. IDFMAD dataset represented in terms of risk of falling and gait velocity groups. a) Stride time and standard deviation; b) stride length and standard deviation

several irregularities. This type of observations allows to relate the quality of the walking pattern with the risk of falling or other health conditions affecting the older adult. Moreover, with a larger set of profiles, it could be useful to identify walking patterns that have already been defined according to different ageing characteristics (e.g. cautious gait, propulsive gait or atasia [11]).

\section{Results}

The gait identification technique introduced in the previous section allows to assess the gait quality of our participants from a spatio-temporal perspective. As before mentioned, we have divided our population by risk of falling and gait velocity. Figures 4 and 5 depict the distribution of the individuals by stride time and length in each pilot and are compared with their standard deviation, i.e. how regular is their pace along the exercise.

Figure 4 represents the IDF and MAD population, were most of the participants had already previous falls or high risk of falling. The distribution in terms of gait velocity is coherent with the risk of falling, since most of the people with high GV belong to the Low risk group. In fact, people in the Low risk group are in general those individuals who better recovered after the 3-months training period in IDF project. The left plot shows that this group is the one with shorter strides in time and higher gait velocity, while the other two risk groups present more variety in results. In addition we observe that the higher the risk of falling is, the more significant is also the standard deviation on time, which is almost proportional to the stride time. This can be interpreted as a sign of irregular pace, which might be due to several stops in the path or an indicator of insecurity. This pattern is also observable in the right side plot, where the stride length is represented. In this case, the distribution of stride length depicts a higher variance in 


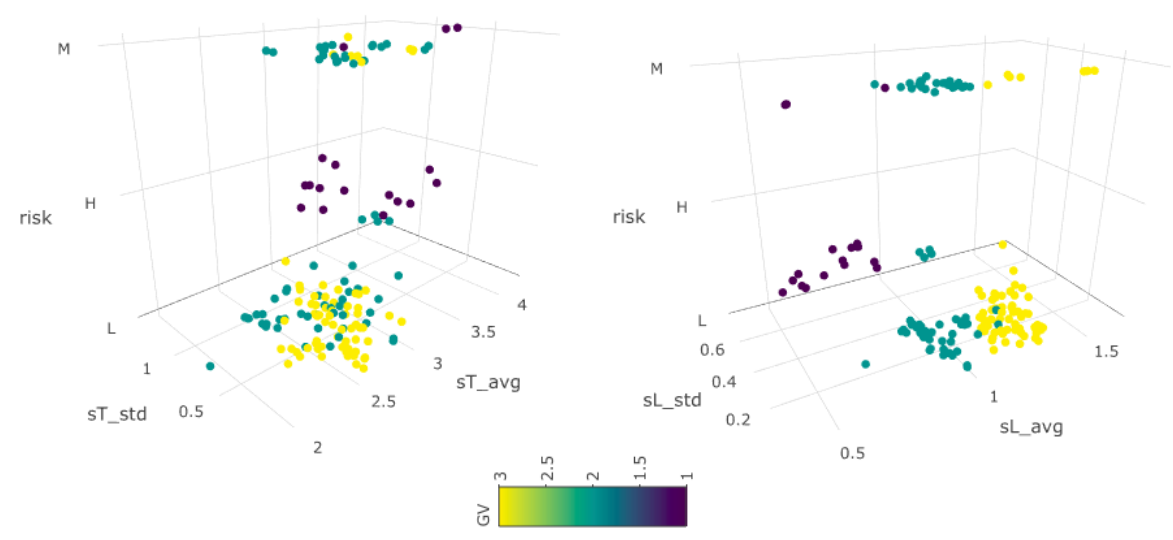

Fig. 5. CVI dataset represented in terms of risk of falling and gait velocity groups. a) Stride time and standard deviation; b) stride length and standard deviation

general, independently of the risk group. This plot shows again a relation between the risk of falling and the spatio temporal features, with a clear separation by gait velocity groups. People at higher risk of falling perform shorter strides and present lower variability. However, the longer the stride is, the higher is the variability too, especially in the case of these individuals at high and medium risk of falling.

The distribution of the CVI participants is depicted in Figure 5, were most of the exercises were performed by individuals aged $<65$ years old. Even though the proportion of young adults $v s$ middle-aged and older participants is quite balanced, the higher representation in exercises is directly related to the gait velocity. In general, young and also middle-aged adults were able to perform 4 to 6 rounds of the $40 \mathrm{~m}$ corridor during the $3 \mathrm{mWT}$ exercise, while the elderly people could only complete 2 rounds in average. The relation between gait velocity, risk of falling and stride length and time is again patent. It is observable that people at high risk and low gait velocity perform shorter strides in length but longer in time. It is also surprising the number of exercises performed by young adults that belong to the second GV group. We were able also to observe that people who have never used a rollator before tend to modify their walking pace and behaviour.

Figure 5 shows that people with low and medium risk of falling are very disperse in terms of stride time. Healthy participants perform longer strides but with a variability of up to one metre. Older individuals with high risk of falling and low gait velocity tend to me more regular in their stride length, but have also an irregular pace. This is probably related to a cautious gait, usually present in geriatric population and sign of fear of falling. We have assumed that gait variability is higher in this dataset due to the length of the exercise, where the fatigue of walking for several minutes is latent in the obtained distribution. 
Finally, if we observe the values in the average stride time in both pilots, we can observe that those in Figure 4 have a larger range of values, going from 0.5 to 3.5 seconds while in Figure 5 these go from 2 to 4 seconds. Values in stride length are more similar, although in the case of IDFMAD pilot there is a bigger group of individuals walking under $0.5 \mathrm{~m} / \mathrm{s}$. This could be helpful to determine different profiles of walking patterns, in which challenged older adults presenting high cadence are performing significantly short strides but in a considerable time, making their ambulation unstable.

In terms of driving skills, results show three main walking behaviours:

- Good laterality and directivity: people who drives around the desired straight line, presenting low oscillations around this trajectory

- Bad laterality, good directivity: individuals that present a deviation and keep this direction during the whole exercise.

- Bad laterality, bad directivity: this scenario presents different situations. In general it represents exercises where the user starts driving to the desired destination but at some point deviates its target goal and changes orientation. Other cases depict multiple changes or corrections of orientation during the exercise.

Since the exercises where performed in indoor scenarios, the most common walking behaviour is the first one, where users where able to maintain a correct orientation with small oscillations, probably due to the swaying movement while walking. The second walking behaviour shows deviations up to 4 metres from the original final point and are in general deviations to the left. This pattern depicts a lack of body force compensation, where people tend to apply more forces from the right side of the body and resulting in this left deviation. Individuals in the third group are those with worse performance in terms of driving skills. However, not all of them belong to high or medium risk of falling.

Figure 6 shows that people with low lateral error has also in general low directivity error. These instances belong to the first walking behaviour group previously described. We can observe that most of the people falling in this group belong to the Low GV and High risk groups. On the opposite, older adults with low risk of falling and walking at high velocity are those with worse driving performances in proportion to the number of instances. The medium risk group is also quite disperse. There are several instances in these two groups with high values of laterality, but mild to moderate directivity errors, whom belong to the second walking behaviour group. It is assumable that people who walk faster also apply larger forces to the $i$-Walker and thus, the laterality decompensation becomes more patent. A more continued testing would be necessary to determine if this pattern is an early detector of physical or cognitive decline in elderly people.

The most critical case is the one representing the third walking behaviour, where not only the lack of body balance is latent, but also the ability to orientate and maintain a given direction. We can observe some instances in the High risk of falling group and in general in the individuals with Low GV distributed in the other risk of falling groups. It is interesting to observe the resulting trajectory of these instances. People with worst directivity results are those that present multiple oscillations along the exercise, even though the laterality is quite maintained (the trajectory is deviated up to $60 \mathrm{~cm}$ approximatively). This walking behaviour corresponds to individuals with a cautious 

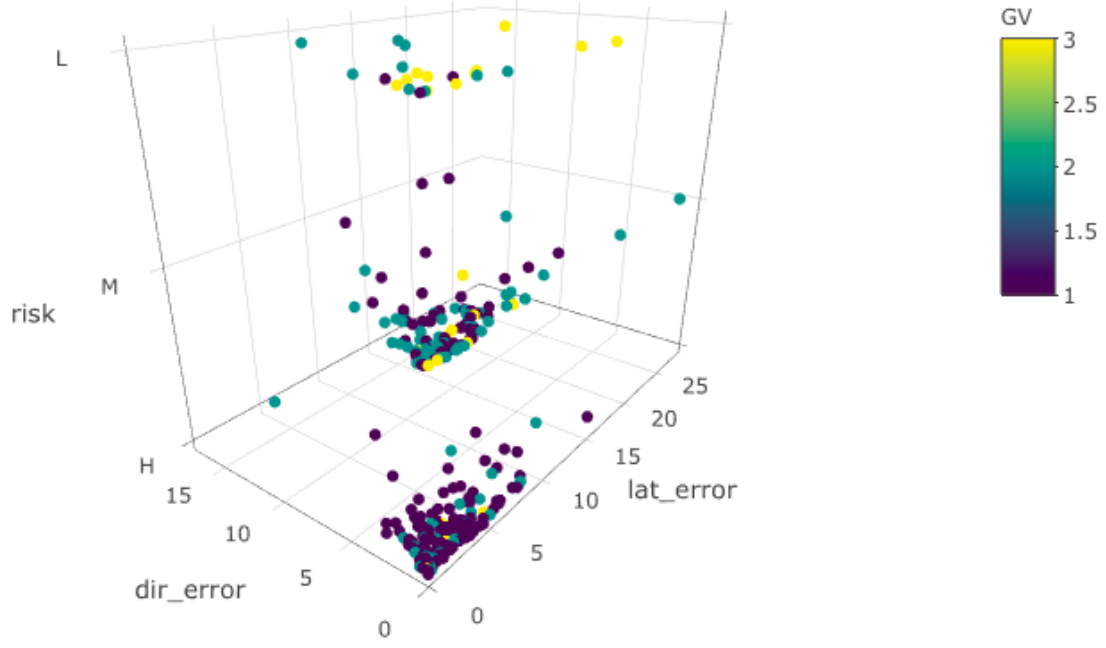

Fig. 6. Relation of the Laterality and Directivity driving skills of the IDFMAD users

and slow gait who are not able to maintain a continuous pace, but instead pauses after each step in order to change the body force from one side to the other.

\section{Conclusion and Future Work}

Demographic statistics show how the elderly population keeps growing as well as their demands. Meeting their needs presents important technical, social and economic challenges. As elderly individuals move toward higher levels of care (i.e., from independent living to assisted living facilities to nursing homes), costs increase while their quality of life rapidly decreases. Assistive Technologies play a key role in nowadays society, since they not only focus on older adults but can also assist younger individuals with some kind of physical or cognitive impairment.

The $i$-Walker provides a better understanding of loading and force distribution for weight support and balance enhancement among users while providing mobility assistance at the same time. In this paper, an approach on spatio-temporal analysis is presented using only the information obtained through the interaction of the participants with the force sensors embedded on the $i$-Walker's handlers. Results show a significant difference in gender, where usually men tend to walk faster and with larger stride length, which is coherent with the anthropometric characteristics. Moreover, there is a relation among age, risk of falling and gait variability. In particular, people performing the walking test at a low cadence, with short and slow strides, are those presenting a higher variance, especially in terms of stride time. Thus, the methodology is able to 
identify those individuals at higher risk of falling or developing other comorbidities. The results obtained are coherent with those found in literature, where gait velocity and cadence are dependent of the gender and age, but also to the risk of falling.

The $i$-Walker' sensor data is also able to generate profiles based on the driving skills of older adults, e.g. if they tend to deviate from their target destination, which can be related to a body force decompensation and, thus, to other early indicators of cognitive decline. A clinical expert should determine which of the walking behaviours above presented is more significant for early detection of decline symptoms: either the lack of orientation or body compensation are clear indicators of dysfunction. A relation between the spatio-temporal parameters and the driving skills has also emerged and presented in [5], where strides are compared based on the force shape obtained with $F_{x} d i f f$ and grouped by similarity using unsupervised learning methods.

Introducing younger participants to our study was useful to confirm this relation, but we consider that this demographic group should not be included in future works. In fact, people that have never used a rollator before tend to modify their walking behaviour, both in terms of gait velocity and force interaction, and might lead to false classifications. An in depth study using specific target pathologies, e.g. frailty or vascular dementia should be necessary in order to further generalize results. Moreover, it could be interesting to add new tools such as a Kinect or video camera in order to verify the sensitivity of the presented methodology.

The main benefit of using sensorized devices is that they are able to provide objective data to clinicians instead of relying on diagnoses obtained by observation, which is the traditional method of physical assessment. By complementing the clinical knowledge with sensor data information, it is expected to design decision support tools able to assist clinicians in the diagnose or rehabilitation treatment, but also to predict the risk of falling in senior population or the development of other commorbidities.

\section{References}

1. E. Adell, S. Wehmhörner, and E. Rydwik. The test-retest reliability of 10 meters maximal walking speed in older people living in a residential care unit. Journal of Geriatric Physical Therapy, 36(2):74-77, 2013.

2. J. Ballesteros, C. Urdiales, A. B. Martinez, and M. Tirado. Automatic assessment of a rollator-user's condition during rehabilitation using the i-walker platform. IEEE Transactions on Neural Systems and Rehabilitation Engineering, 25(11):2009-2017, 2017.

3. A. Cortés, C. Barrué, J. Moreno, F. Barban, M. Melideo, U. Cortés, and R. Annicchiarico. A fall prevention protocol using the i-walker robotic rollator: The i-dont-fall project. In 14th International Conference on Mobility and Transport for Elderly and Disabled Persons, TRANSED 2015, Lisbon, Portugal, 2015, pages 1366-1380, 2015.

4. A. Cortés, A. B. Martínez, and J. Béjar, editors. 4th Workshop on ICTs for improving Patients Rehabilitation Research Techniques (REHAB '16), New York, NY, USA, 2016. ACM.

5. A. Cortés, M. Ojeda, and A. B. Béjar, Javier Martínez. An approach to gait analysis from human-rollator interaction: The $i$-walker. $21^{\text {th }}$ International Conference of the Catalan Association for Artificial Intelligence, Spain, October 8-10, 2018, 2018.

6. U. Cortés, A. Martínez-Velasco, C. Barrué, and R. Annicchiarico. AI based fall management services - the role of the $i$-Walker in I-DONTFALL. In Advances in Artificial Intelligence 
- $11^{\text {th }}$ Mexican International Conference on Artificial Intelligence, MICAI 2012, San Luis Potosí, Mexico, 2012., pages 395-406, 2012.

7. Integrated prevention and Detection sOlutioNs Tailored to the population and Risk Factors associated with FALLs. http://www.idontfall.eu/, 2010.

8. M. Montero-Odasso, M. Schapira, E. R. Soriano, M. Varela, R. Kaplan, L. A. Camera, and L. M. Mayorga. Gait velocity as a single predictor of adverse events in healthy seniors aged 75 years and older. The Journals of Gerontology Series A: Biological Sciences and Medical Sciences, 60(10):1304-1309, 2005.

9. M. Montero-Odasso, J. Verghese, O. Beauchet, and J. M. Hausdorff. Gait and cognition: A complementary approach to understanding brain function and the risk of falling. Journal of American Geriatrics, 60(11):2127-2136, 2012.

10. C. Nooijen, N. ter Hoeve, and E. Field-Fote. Gait quality is improved by locomotor training in individuals with sci regardless of training approach. Journal of NeuroEngineering and Rehabilitation, 6(36), 2009.

11. W. Pirker and R. Katzenschlager. Gait disorders in adults and the elderly. Wiener Klinische Wochenschrift, 129(3-4):81-95, 2017.

12. C. Prakash, R. Kumar, and N. Mittal. Recent developments in human gait research: parameters, approaches, applications, machine learning techniques, datasets and challenges. Artificial Intelligence Review, pages 1-40, 2016.

13. H. Shimada, A. Tiedemann, S. Lord, M. Suzukawa, H. Makizako, K. Kobayashi, and T. Suzuki. Physical factors underlying the association between lower walking performance and falls in older people: A structural equation model. Archives of gerontology and geriatrics, 53(2):131-134, 2010.

14. M. E. Tinetti, M. Speechley, and S. F. Ginter. Risk factors for falls among elderly persons living in the community. New England journal of medicine, 319(26):1701-1707, 1988.

15. C. Urdiales. Collaborative Assistive Robot for Mobility Enhancement (CARMEN) - The bare necessities: Assisted wheelchair navigation and beyond, volume 27 of Intelligent Systems Reference Library. Springer, 2012.

16. T. Wang, J.-P. Merlet, G. Sacco, P. Robert, J.-M. Turpin, B. Teboul, A. Marteu, and O. Guerin. Walking analysis of young and elderly people by using an intelligent walker \{ANG\}. Robotics and Autonomous Systems, pages -, 2014.

17. WHO. European health report 2012: charting the way to well-being. World Health Organization, 2013.

18. A. Yorozu, T. Moriguchi, and M. Takahashi. Improved leg tracking considering gait phase and spline-based interpolation during turning motion in walk tests. Sensors, 15(9):22451, 2015. 